\title{
Alkyl Chain Barriers for Kinetic Optimization in Dye-Sensitized Solar Cells
}

\author{
Jessica E. Kroeze $,^{*},+, \S$ Narukuni Hirata, ${ }^{\dagger}$ Sara Koops, ${ }^{\dagger}$ Md. K. Nazeeruddin, ${ }^{\ddagger}$ \\ Lukas Schmidt-Mende, ${ }^{\ddagger}$ Michael Grätzel, ${ }^{\ddagger}$ and James R. Durrant ${ }^{*}$, \\ Contribution from the Centre for Electronic Materials and Devices, Imperial College London, \\ London SW7 2AY, United Kingdom, and Laboratory for Photonics and Interfaces, Institute of \\ Chemical Science and Engineering, Swiss Federal Institute of Technology,
}

CH-1015 Lausanne, Switzerland

\begin{abstract}
The optimization of interfacial charge transfer is crucial to the design of dye-sensitized solar cells. In this paper we address the dynamics of the charge separation and recombination in liquid-electrolyte and solid-state cells employing a series of amphiphilic ruthenium dyes with varying hydrocarbon chain lengths, acting as an insulating barrier for electron-hole recombination. Dynamics of electron injection, monitored by time-resolved emission spectroscopy, and of charge recombination and regeneration, monitored by transient optical absorption spectroscopy, are correlated with device performance. We find that increasing dye alkyl chain length results in slower charge recombination dynamics to both the dye cation and the redox electrolyte or solid-state hole conductor (spiro-OMeTAD). These slower recombination dynamics are however paralleled by reduced rates for both electron injection into the $\mathrm{TiO}_{2}$ electrode and dye regeneration by the $\mathrm{I}^{-} / \mathrm{I}_{3}^{-}$redox couple or spiro-OMeTAD. Kinetic competition between electron recombination with dye cations and dye ground state regeneration by the iodide electrolyte is found to be a key factor for liquid electrolyte cells, with optimum device performance being obtained when the dye regeneration is just fast enough to compete with electron-hole recombination. These results are discussed in terms of the minimization of kinetic redundancy in solid-state and liquid-electrolyte dye-sensitized photovoltaic devices.
\end{abstract}

\section{Introduction}

During the past decade, dye-sensitized solar cells (DSSCs) have attracted widespread interest as a promising, low-cost alternative to conventional silicon photovoltaic devices. ${ }^{1,2}$ Such DSSCs consist of a nanocrystalline mesoporous metal oxide (typically $\mathrm{TiO}_{2}$ ) film, coated with a monolayer of a sensitizer dye, interpenetrated by a hole-transporting medium (HTM), such as an $\mathrm{I}^{-} / \mathrm{I}_{3}{ }^{-}$redox liquid-electrolyte or solid-state molecular hole conductor. The power conversion efficiency of such devices is strongly dependent upon the optimization of electron-transfer dynamics at the metal oxide/dye/HTM interface, as illustrated in Figure 1. In particular, high device output voltages are dependent upon the minimization of interfacial charge recombination losses (CR1 and CR2 in Figure 1). ${ }^{3}$ Several groups have previously investigated the potential of inserting barrier layers to retard such recombination losses. ${ }^{4-11}$ In this paper,

\footnotetext{
$\doteqdot$ Imperial College London.

$\$$ Swiss Federal Institute of Technology.

$\S$ Present address: Molecular Materials and Nanosystems, Laboratory of Macromolecular and Organic Chemistry, Eindhoven University of Technology, P.O. Box 513, 5600 MB Eindhoven, The Netherlands.

(1) Hagfeldt, A.; Grätzel, M. Acc. Chem. Res. 2000, 33, 269-277.

(2) Grätzel, M. Nature 2001, 414, 338-344.

(3) Huang, S. Y.; Schlichthorl, G.; Nozik, A. J.; Gratzel, M.; Frank, A. J. J. Phys. Chem. B 1997, 101, 2576-2582.

(4) Tennakone, K.; Kumara, G.; Kottegoda, I. R. M.; Perera, V. P. S. Chem. Commun. 1999, 15-16.
}

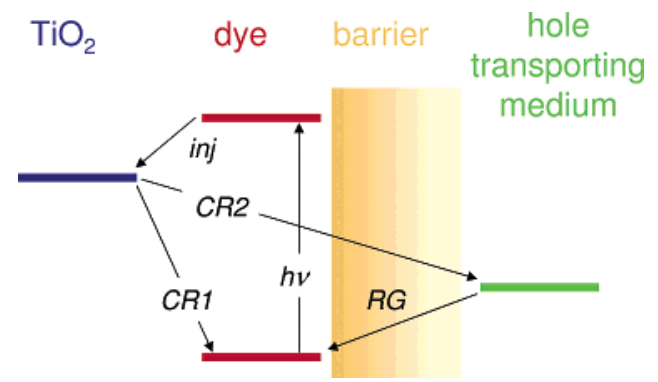

Figure 1. Schematic representation of the various components of a dyesensitized nanocrystalline semiconductor solar cell: a ruthenium bipyridyl dye-sensitized nanocrystalline $\mathrm{TiO}_{2}$ film, interpenetrated by a liquid $\mathrm{I}^{-} / \mathrm{I}_{3}{ }^{-}$ redox electrolyte or a solid-state spiro-OMeTAD hole conducting matrix containing lithium salts and 4-tert-butyl pyridine additives. Also shown are the different electron-transfer processes in the solar cell: electron injection from dye excited state into the conduction band of $\mathrm{TiO}_{2}$ semiconductor (inj); the subsequent regeneration of the dye by electron transfer to the dye cation from the hole transporting medium (RG); charge recombination to the cation of the dye (CR1) and recombination to the hole transporting medium (CR2). The shaded area depicts the charge-transfer blocking function of the hydrophobic alkyl chains of the dye.

we focus on the modulation of electron-transfer dynamics achieved by one such strategy, namely the addition of alkyl chains to the sensitizer dye, aimed at insertion of a barrier layer between the sensitizer dye and the HTM (see Figure 1), and their impart upon device performance. 
The control of interfacial charge-transfer processes is of central importance to the design of DSSCs. ${ }^{12-17}$ For example, a high open-circuit voltage $\left(V_{\mathrm{oc}}\right)$ requires the minimization of interfacial recombination losses, whereas high charge separation yields are necessary to achieve high short-circuit photocurrent densities $\left(J_{\mathrm{sc}}\right)$. Previous studies have shown that charge recombination of $\mathrm{TiO}_{2}$ electrons with the oxidized HTM (CR2 in Figure 1) is the key factor limiting $V_{\mathrm{oc}}$ in both liquid-electrolyte and solid-state DSSCs. Charge recombination to the dye cation, CR1 in Figure 1, is in kinetic competition with the desired regeneration reaction, $\mathrm{RG}$, and therefore limits the charge separation yield. Strategies directed toward the minimization of interfacial recombination losses have included not only the insertion of metal oxide blocking layers ${ }^{7,18}$ but also energetic redox cascades for multistep hole transfer, ${ }^{19}$ as well as supermolecular sensitizer dyes..$^{20-22}$ We have recently demonstrated that the optimization of electron-transfer dynamics in DSSCs requires the minimization of "kinetic redundancy" for the charge separation steps. ${ }^{23}$ This strategy is based upon the appreciation that the kinetics of charge separation and charge recombination in a DSSC are often temporally correlated, with changes to the device design intended to achieve slower recombination dynamics often resulting in a parallel retardation of charge separation. In our previous study we focused on the correlation between electron injection dynamics from the excited sensitizer dye into the metal oxide and the charge recombination to a liquid redox electrolyte as a function of $\mathrm{TiO}_{2}$ surface charge.

It was demonstrated that optimum device performance was obtained when the electron injection dynamics were just fast enough to compete successfully with the dye excited-state decay, corresponding to an injection half time of 150 ps. Such moderately "slow" charge separation dynamics still resulted in a high electron injection yield, while allowing interfacial charge recombination losses to be minimized.

In this paper we focus our studies toward addressing the extent of kinetic redundancy in the dye regeneration reaction (process RG in Figure 1) in liquid-electrolyte and solid-state

(5) Zaban, A.; Chen, S. G.; Chappel, S.; Gregg, B. A. Chem. Commun. 2000 , $2231-2232$.

(6) Gregg, B. A.; Pichot, F.; Ferrere, S.; Fields, C. L. J. Phys. Chem. B 2001, $105,1422-1429$.

(7) Palomares, E.; Clifford, J. N.; Haque, S. A.; Lutz, T.; Durrant, J. R. J. Am. Chem. Soc. 2003, 125, 475-482.

(8) Clifford, J. N.; Palomares, E.; Nazeeruddin, K.; Thampi, R.; Grätzel, M.; Durrant, J. R. J. Am. Chem. Soc. 2004, 126, 5670-5671

(9) Ito, S.; Makari, Y.; Kitamura, T.; Wada, Y.; Yanagida, S. J. Mater. Chem. 2004, 14, 385-390.

(10) Schmidt-Mende, L.; Zakeeruddin, S. M.; Grätzel, M. Appl. Phys. Lett. 2005 86.

(11) Yum, J. H.; Nakade, S.; Kim, D. Y.; Yanagida, S. J. Phys. Chem. B 2006, 110, 3215-3219.

(12) Bauer, C.; Boschloo, G.; Mukhtar, E.; Hagfeldt, A. J. Phys. Chem. B 2002, 106, 12693-12704.

(13) Durrant, J. R.; Haque, S. A.; Palomares, E. Coord. Chem. Rev. 2004, 248, 1247-1257.

(14) Anderson, N. A.; Lian, T. Q. Ann. Rev. Phys. Chem. 2005, 56, 491-519.

(15) Meyer, G. J. Inorg. Chem. 2005, 44, 6852-6864.

(16) Tributsch, H. C.R. Chim. 2006, 9, 584-596.

(17) Cazzanti, S.; Caramori, S.; Argazzi, R.; Elliot, C. M.; Bignozzi, C. A. J. Am. Chem. Soc. 2006, 9996-9997.

(18) Kay, A.; Grätzel, M. Chem. Mater. 2002, 14, 2930-2935.

(19) Hirata, N.; Kroeze, J. E.; Park, T.; Jones, D.; Haque, S. A.; Holmes, A. B.; Durrant, J. R. Chem. Commun. 2006, 535-537.

(20) Hirata, N.; Lagref, J. J.; Palomares, E. J.; Durrant, J. R.; Nazeeruddin, M. K.; Grätzel, M.; Di Censo, D. Chem.-Eur. J. 2004, 10, 595-602.

(21) Haque, S. A.; Handa, S.; Peter, K.; Palomares, E.; Thelakkat, M.; Durrant, J. R. Angew. Chem., Int. Ed. 2005, 44, 5740-5744.

(22) Snaith, H. J.; Zakeeruddin, S. M.; Schmidt-Mende, L.; Klein, C.; Grätzel, M. Angew. Chem., Int. Ed. 2005, 22, 2-5.

(23) Haque, S. A.; Palomares, E.; Cho, B. M.; Green, A. N. M.; Hirata, N.; Klug, D. R.; Durrant, J. R. J. Am. Chem. Soc. 2005, 127, 3456-3462.

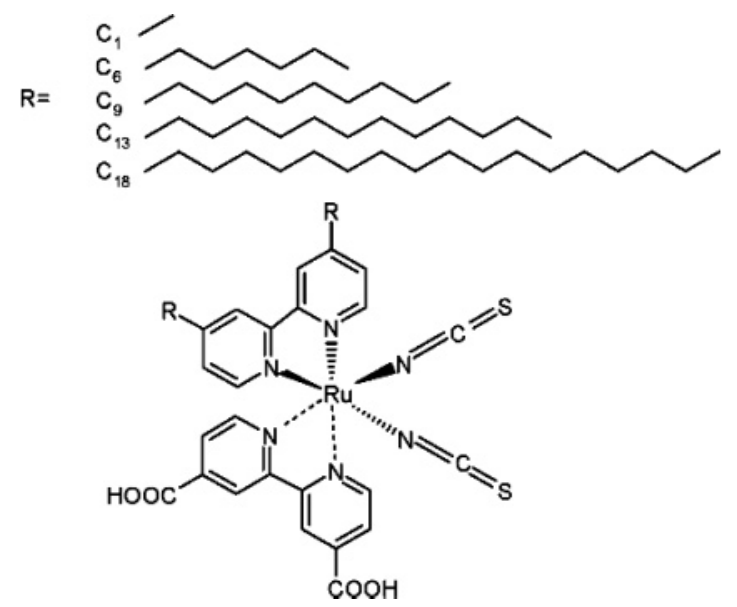

Figure 2. Chemical structure of the amphiphilic sensitizer dyes used in this study.

DSSCs. This reaction is a key in the function of these devices but has received remarkably little attention to date. The experimental strategy is based upon employing transient absorption spectroscopy to monitor the transient dynamics of a series of amphiphilic ruthenium bipyridyl sensitizer dyes substituted with alkyl chains of various lengths. The structures of the dyes employed are shown in Figure 2. These dyes were originally developed with the intention that the alkyl chains would form a hydrophobic layer over the sensitizer dye layer in order to protect the dye layer against water ingression from the electrolyte. The validity of this concept was proven by stability studies of device performance, showing improved device stability over a 40-day trial period. ${ }^{24}$ Subsequent studies have demonstrated that such alkyl-substituted dyes are particularly suited for DSSCs employing ionic-liquid and spiro-OMeTAD HTMs. ${ }^{10,25,26}$ In this paper we focus on the ability of such alkyl chains to function as an electrically insulating barrier, thereby reducing interfacial charge recombination losses in the DSSCs.

In liquid electrolyte DSSCs employing the most widely used sensitizer dye, $\mathrm{RuL}_{2}(\mathrm{NCS})_{2}$ ( $\mathrm{L}=4,4^{\prime}$-dicarboxy-2,2'-bipyridyl), regeneration of the dye ground state by electron transfer from the liquid electrolyte (RG) exhibits a half time of $\sim 1 \mu \mathrm{s} .{ }^{27} \mathrm{In}$ typical redox electrolytes, this regeneration reaction competes efficiently with charge recombination of injected electrons with dye cations, CR1, which is typically on the $10 \mu \mathrm{s}-1 \mathrm{~ms}$ time scale dependent upon the position of the $\mathrm{TiO}_{2}$ Fermi level. ${ }^{28}$ As such, $k_{\mathrm{RG}}>k_{\mathrm{CR} 1}$, and the regeneration reaction exhibits modest kinetic redundancy, suggesting that a limited retardation of the regeneration dynamics resulting from the insertion of a small physical barrier between the dye and the electrolyte might reduce recombination losses due to CR2 without significantly reducing the efficiency of the regeneration reaction. For solidstate DSSCs employing the molecular hole conductor $2,2^{\prime}-7,7^{\prime}-$ tetrakis( $N, N$-di-p-methoxyphenylamine) $9,9^{\prime}$-spirobifluorene (spiro-

(24) Zakeeruddin, S. M.; Nazeeruddin, M. K.; Humphry-Baker, R.; Pechy, P.; Quagliotto, P.; Barolo, C.; Viscardi, G.; Grätzel, M. Langmuir 2002, 18 , 952-954.

(25) Schmidt-Mende, L.; Kroeze, J. E.; Durrant, J. R.; Nazeeruddin, M. K. Grätzel, M. Nano Lett. 2005, 5, 1315-1320.

(26) Nazeeruddin, M. K.; Zakeeruddin, S. M.; Lagref, J. J.; Liska, P.; Comte, P.; Barolo, C.; Viscardi, G.; Schenk, K.; Graetzel, M. Coord. Chem. Rev. 2004, 248, 1317-1328.

(27) Montanari, I.; Nelson, J.; Durrant, J. R. J. Phys. Chem. B 2002, 106, $12203-$ 12210.

(28) Nelson, J.; Haque, S. A.; Klug, D. R.; Durrant, J. R. Phys. Rev. B 2001, 6320 , art. no 205321 
OMeTAD), the regeneration is 3 orders of magnitude faster $\left(t_{50 \%}\right.$ $<1 \mathrm{~ns}),{ }^{29}$ indicating an even greater potential to minimize kinetic redundancy in the device by the insertion of such physical barrier layers. Indeed, we have recently demonstrated that the addition of alkyl chains to the molecular sensitizer can indeed retard recombination dynamics in solid-state DSSCs, resulting in a marked increase of the $V_{\mathrm{oc}}$ and overall device performance. $^{25}$

In this paper, we employ transient absorption and emission spectroscopies to monitor the dynamics of charge separation and charge recombination in both liquid-electrolyte and solidstate DSSCs employing the sensitizer dye series shown in Figure 2. We investigate the extent to which the addition of alkyl chains to the sensitizer dye can minimize kinetic redundancy in such DSSCs and demonstrate how such minimization correlates with improvements in photovoltaic device efficiency.

\section{Experimental Section}

Chemical Compounds. The amphiphilic sensitizer dyes $\mathrm{Ru}\left(\mathrm{H}_{2-}\right.$ dcbpy)(4,4'-dimethyl-2,2'-bipyridine)(NCS $)_{2}$ (C1 dye), $\mathrm{Ru}\left(\mathrm{H}_{2} \mathrm{dcbpy}\right)-$ (4,4'-dihexyl-2,2'-bipyridine)(NCS $)_{2}$ (C6 dye), $\mathrm{Ru}\left(\mathrm{H}_{2} \mathrm{dcbpy}\right)\left(4,4^{\prime}\right.$-nonyl$2,2^{\prime}$-bipyridine $)(\mathrm{NCS})_{2}$ (C9 dye), $\mathrm{Ru}\left(\mathrm{H}_{2} \mathrm{dcbpy}\right)\left(4,4^{\prime}\right.$-tridecyl-2,2' bipyridine $)(\mathrm{NCS})_{2} \quad(\mathbf{C 1 3}$ dye $), \quad \mathrm{Ru}\left(\mathrm{H}_{2}\right.$ dcbpy $)\left(4,4^{\prime}\right.$-octadecyl-2,2'bipyridine $)(\mathrm{NCS})_{2}\left(\mathrm{C18}\right.$ dye), and $(\mathrm{Bu} 4 \mathrm{~N})_{2}\left[\mathrm{Ru}(\mathrm{dcbpyH})_{2}(\mathrm{NCS})_{2}\right](\mathrm{N719})$ were synthesized as published previously. ${ }^{24,26,30}$

TCO-coated glass substrates were obtained from Hartford Glass, USA ( $15 \Omega / \mathrm{cm}^{2}$ F-doped $\mathrm{SnO}_{2}$ ). The $\mathrm{TiO}_{2}$ paste, consisting of $10-$ 15-nm-sized anatase particles, was prepared via a sol-gel route, as described previously. ${ }^{31}$ Spiro-OMeTAD was supplied by Avecia, Manchester (now Merck Chemicals Ltd.) and was $>99.5 \%$ pure. All solvents were purchased from Aldrich and were HPLC grade.

Sample/Device Preparation. Solid-state and liquid-electrolyte device preparation have been described in detail previously. ${ }^{25}$ Briefly, after cleaning F-doped $\mathrm{SnO}_{2}$ glass substrates (with acetone, methanol, and Helmanex), an $\sim 100 \mathrm{~nm}$ compact layer of $\mathrm{TiO}_{2}$ was deposited by spray-pyrolysis. A nanocrystalline, mesoporous $\mathrm{TiO}_{2}$ film, thickness 2 $\mu \mathrm{m}$, was fabricated via doctor-blading the $\mathrm{TiO}_{2}$ paste, followed by sintering at $450{ }^{\circ} \mathrm{C}$. Dyes were adsorbed to the $\mathrm{TiO}_{2}$ film by immersion overnight in a $0.3 \mathrm{mM}$ solution in acetonitrile/tert-butanol (1:1) and subsequently carefully rinsed with acetonitrile.

Transparent counter electrodes were prepared by chemically depositing platinum from $0.05 \mathrm{M}$ hexachloroplatinic acid in 2-propanol onto a second slide of conducting glass. Sandwich cells $\left(1 \mathrm{~cm}^{2}\right)$ were then prepared by sealing together the $\mathrm{TiO}_{2}$-coated electrode with the counter electrode using a transparent film of Surlyn 1472 polymer (DuPont Ltd.) at $110{ }^{\circ} \mathrm{C}$. The redox electrolyte, consisting of $0.60 \mathrm{M}$ butylmethyl imidazolium iodide, $0.03 \mathrm{M} \mathrm{I}_{2}, 0.10 \mathrm{M}$ guanidinium thiocyanate, and $0.50 \mathrm{M}$ 4-tert-butylpyridine $(t \mathrm{BP})$ in a mixture of acetonitrile and valeronitril (volume ratio: 85:15), was then introduced through holes drilled in the counter electrode, which were sealed immediately with microscope cover slides and additional strips of Surlyn to avoid leakage.

For the preparation of solid-state devices, the hole conductor matrix was applied by depositing a solution of Spiro-OMeTAD $(0.17 \mathrm{M}$ in chlorobenzene, also containing $0.025 \mathrm{mM}$ lithium bis-trifluorosulfonimide $\left(\mathrm{Li}\left[\mathrm{CF}_{3} \mathrm{SO}_{2}\right]_{2} \mathrm{~N}, \mathrm{Li}^{+}\right), 0.13 \mathrm{M} t \mathrm{BP}$, and $0.3 \mathrm{mM}$ tris(4-bromophenyl) ammoniumylhexachloroantimonate dopant) on the dye-coated substrate, allowing the HTM to penetrate the film during $1 \mathrm{~min}$, followed by a spin-coating step at $1800 \mathrm{rpm}$. Samples were stored in

(29) Bach, U.; Tachibana, Y.; Moser, J. E.; Haque, S. A.; Durrant, J. R.; Grätzel, M.; Klug, D. R. J. Am. Chem. Soc. 1999, 121, 7445-7446.

(30) Nazeeruddin, M. K.; Zakeeruddin, S. M.; Humphry-Baker, R.; Jirousek, M.; Liska, P.; Vlachopoulos, N.; Shklover, V.; Fischer, C. H.; Grätzel, M. Inorg. Chem 1999, 38, 6298-6305.

(31) Barbe, C. J.; Arendse, F.; Comte, P.; Jirousek, M.; Lenzmann, F.; Shklover, V.; Grätzel, M. J. Am. Ceram. Soc. 1997, 80, 3157-3171.

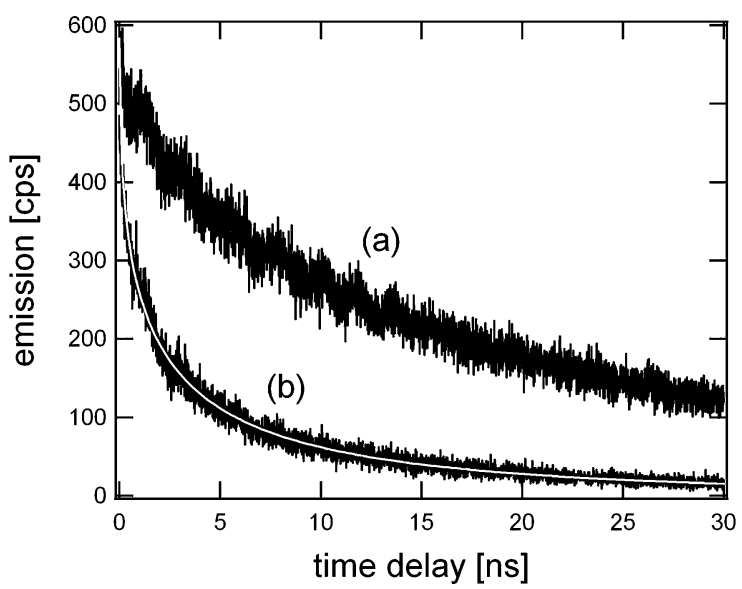

Figure 3. Time-resolved emission decays for C9-sensitized $\mathrm{ZrO}_{2}$ (a) and $\mathrm{TiO}_{2}$ (b) films covered in an inert solvent. Also shown is the stretchedexponential fit to the $\mathrm{TiO}_{2}$ data (smooth white line). Data collected for matched sample optical densities and data collection times, allowing direct comparison of the magnitudes of the emission transients.

the dark under a vacuum prior to laser transient spectroscopy and/or gold evaporation.

Functional Characterization. Electron injection dynamics were monitored using time-correlated single photon counting (TCSPC), employing a Jobin Yvon IBH Fluorocube laser system. The apparatus employed $467 \mathrm{~nm}$ excitation (1 $\mathrm{MHz}$ repetition rate, instrument response $250 \mathrm{ps}$ fwhm), with a $715 \mathrm{~nm}$ high pass filter for emission detection. Samples consisted of dye-sensitized electrodes covered in either 3-methoxypropionitrile (inert solvent) or a redox electrolyte consisting of $0.1 \mathrm{M} \mathrm{I}_{2}, 0.1 \mathrm{M}$ lithium iodide, $0.6 \mathrm{M}$ tetrabutylammonium iodide, and $0.50 \mathrm{M}$ tert-butylpyridine in 3-methoxypropionitrile solvent. Transient absorption experiments were conducted as reported previously, ${ }^{32}$ employing low-intensity pulses (532 nm, $\sim 40 \mu \mathrm{J} / \mathrm{cm}^{2} /$ pulse) from a nitrogen-laser-pumped dye laser (PTI GL-3300, 0.8 Hz, 600 ps pulse duration) or a Nd:YAG laser (Quantel Big Sky Ultra CFR, $1 \mathrm{~Hz}$, $6 \mathrm{~ns}$ pulse duration) and using home-built photodiode-based optical amplification and filtering equipment. Transient absorption studies of the dye-sensitized nanocrystalline films only were performed by covering the film with a 1:1 ethylene carbonate/propylene carbonate solution (inert solvent) and glass cover slide. Current-voltage characteristics were measured using a Keithley 2400 Source Meter and a $1000 \mathrm{~W}$ Oriel solar simulator fitted with an AM1.5 global filter set as the light source. Light intensity was changed with meshes in front of the light source and measured with a $\mathrm{Si}$ photodiode and calibrated to $1000 \mathrm{~W} / \mathrm{m}^{2}$ for 1 sun.

\section{Results}

Electron Injection. We consider first transient emission studies of electron injection dynamics for this dye series. Typical data are shown in Figure 3, including control data employing a C9-sensitized nanocrystalline $\mathrm{ZrO}_{2}$ film and data for C9sensitized $\mathrm{TiO}_{2}$ films covered in inert solvent. $\mathrm{ZrO}_{2}$ was employed for the control data as its high conduction band edge prevents electron injection from the dye excited state. ${ }^{33,34}$ For this control film, a biphasic decay is observed, with lifetimes (amplitudes) of $3.3 \mathrm{~ns}(0.05)$ and $21.5 \mathrm{~ns}(0.95)$. Note that these data were collected under aerobic conditions and, therefore, represent an upper (fastest) limit for the rate of excited-state

(32) Haque, S. A.; Park, T.; Holmes, A. B.; Durrant, J. R. ChemPhysChem 2003 4, 89-93.

(33) Kay, A.; Humphrybaker, R.; Grätzel, M. J. Phys. Chem. 1994, 98, 952959

(34) Tachibana, Y.; Moser, J. E.; Grätzel, M.; Klug, D. R.; Durrant, J. R. J. Phys. Chem. 1996, 100, 20056-20062. 
Table 1. Emission Decay Half Times $\left(\tau_{\mathrm{inj}}\right)$ for the Dye Series

\begin{tabular}{llcc}
\hline & \multicolumn{3}{c}{ emission decay half times $(\mathrm{ps})$} \\
\cline { 2 - 4 } dye & $\mathrm{TiO}_{2}$ with solvent $^{\mathrm{a}}$ & $\mathrm{TiO}_{2}$ with electrolyte $^{\mathrm{a}}$ & $\mathrm{ZrO}_{2}$ with solvent $^{b}$ \\
\hline C1 & $<60$ & $625 \pm 50$ & $4000 \pm 100$ \\
C6 & $210 \pm 100$ & $2000 \pm 150$ & $7000 \pm 750$ \\
C9 & $1000 \pm 200$ & $2600 \pm 200$ & $10000 \pm 2000$ \\
C13 & $<60$ & $1750 \pm 150$ & $5900 \pm 250$ \\
C18 & $220 \pm 50$ & $2500 \pm 400$ & $3000 \pm 500$ \\
N719 & $<60$ & $150 \pm 50$ & $11800 \pm 500$ \\
\hline
\end{tabular}

${ }^{a}$ Half times obtained from stretched-exponential fits to the transient decays after convolution of the instrument response, as shown in Figure 3 , fixing the initial amplitude of the fit to the initial amplitude of the $\mathrm{ZrO}_{2}$ data. Further details will be presented elsewhere (SK, JRD, in preparation). ${ }^{b}$ Half times obtained from biexponential fits to the transient decays after convolution of the instrument response.

decay to the ground state in a sealed, and therefore anaerobic, DSSC. For the $\mathrm{TiO}_{2}$ films covered in inert solvent, this emission is strongly quenched, consistent with efficient electron injection from the dye excited state. ${ }^{34}$ Note that these data are collected with matched numbers of absorbed photons, allowing direct comparison of the amplitudes of emission decays. The observation that the initial amplitude of the $\mathbf{C 9} / \mathrm{TiO}_{2}$ emission decay is similar to that of the control $\mathbf{C} / / \mathrm{ZrO}_{2}$ decay is indicative of negligible electron injection much faster than the 200 ps instrument response. Rather the emission decay for this dye shows a half time of 1000 ps, indicating significantly slower electron injection dynamics into $\mathrm{TiO}_{2}$ than those for the more widely used $\mathrm{Ru}(\mathrm{dcbpy})_{2}(\mathrm{NCS})_{2}$ dye.

For all sensitizer dyes, emission decay dynamics for sensitized $\mathrm{TiO}_{2}$ films exhibit single stretched-exponential behavior (int $\propto$ $\left.\exp \left[-(t / \tau)^{\alpha}\right]\right)$, as illustrated by the fit to the decay shown in Figure 3. Such stretched-exponential behavior is consistent with inhomogeneous broadening of the energetics of electron injection, as we have discussed in detail previously. ${ }^{35}$ Table 1 shows emission decay half times obtained from data such as that shown in Figure 3 for dye series $\mathbf{C 1}$ through C18. For comparison, this table also shows half times obtained by analogous emission decays for the $\mathrm{Ru}(\mathrm{dcbpy})_{2}(\mathrm{NCS})_{2}$ tetrabutyl ammonium salt (N719) dye, which show good agreement with our previous ultrafast transient absorption studies with this sensitizer dye. Compared to the data for $\mathrm{TiO}_{2}$ films covered in inert solvent, for all dyes the decay dynamics are significantly slower in the presence of a redox electrolyte, with the half time increased on average by an order of magnitude, consistent with our previous ultrafast transient absorption studies for $\mathrm{Ru}(\mathrm{dcbpy})_{2}(\mathrm{NCS})_{2}$ sensitized films. ${ }^{23}$ For the dye series $\mathbf{C 1}$ up to $\mathbf{C 9}$, it is apparent that increasing the alkyl chain length results in a significant slowing of emission decay half times, indicative of slower injection dynamics. Dyes C13 and C18 do not follow this trend, as we discuss further below. For the $\mathbf{C 1 8}$ dye, the emission decay dynamics observed for the $\mathrm{TiO}_{2}$ film in the presence of a redox electrolyte were similar to those observed for the control $\mathrm{ZrO}_{2}$ films, indicating that electron injection for this dye may not compete effectively with excited-state decay to ground. This observation is consistent with our previous observation, by transient absorption spectroscopy, of a reduced electron injection yield with this dye. This reduced injection yield has been ascribed previously to a collapse of the long $\mathbf{C 1 8}$ alkyl chains, resulting in inhomogeneous adsorption to the $\mathrm{TiO}_{2}$ surface. ${ }^{25}$

(35) Tachibana, Y.; Haque, S. A.; Mercer, I. P.; Moser, J. E.; Klug, D. R.; Durrant, J. R. J. Phys. Chem. B 2001, 105, 7424-7431.

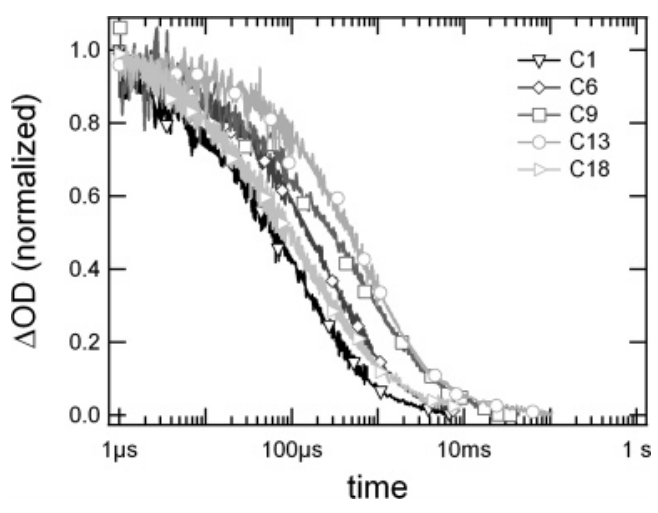

Figure 4. Transient absorption data monitoring electron recombination with dye cations $(\mathrm{CR} 1)$ at $800 \mathrm{~nm}$, in $\mathrm{TiO}_{2}$ films sensitized by the $\mathbf{C 1}-\mathbf{C 1 8}$ dye series covered in inert solvent. For the purpose of comparison, all transients have been normalized to unity.

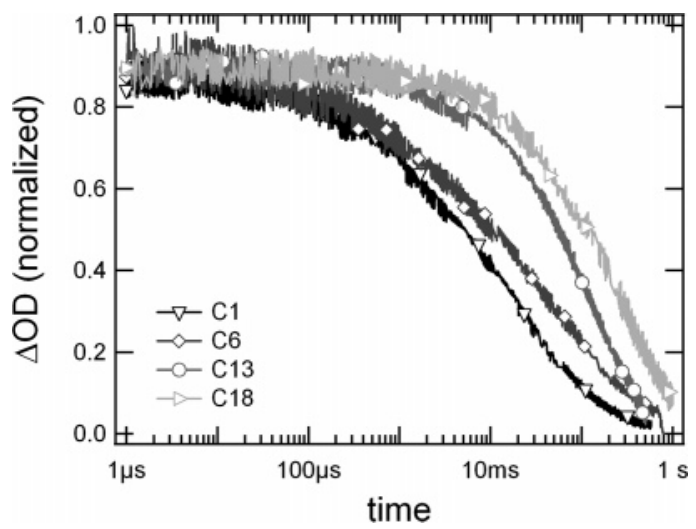

Figure 5. Transient absorption data monitoring electron recombination to the $\mathrm{I}^{-} / \mathrm{I}_{3}-$ redox electrolyte at $1000 \mathrm{~nm}$, in liquid-electrolyte DSSCs employing the C1-C18 sensitizer dye series. For the purpose of comparison, all transients have been normalized to unity.

Charge Recombination (CR1 and CR2). We first consider the dynamics of charge recombination of injected electrons with dye cations observed for sensitized $\mathrm{TiO}_{2}$ films in the absence of a redox electrolyte or hole-conducting medium (CR1 in Figure 1). These dynamics were monitored by transient absorption spectroscopy as previously by probing the decay of the dye cation absorption band at $800 \mathrm{~nm} .{ }^{34}$ Typical data are shown in Figure 4. All decay dynamics exhibit stretched-exponential behavior $\left(\triangle \mathrm{OD} \propto \exp \left[-(t / \tau)^{\alpha}\right]\right)$, characteristic of recombination dynamics being primarily controlled by electron trapping/ detrapping dynamics in the $\mathrm{TiO}_{2}$ particles. ${ }^{28}$ An $\sim 5$-fold retardation of the $\mathrm{CR} 1$ recombination half time is observed with increasing alkyl chain length up to $\mathbf{C 1 3}$, from $\sim 90$ to $490 \mu \mathrm{s}$.

We now turn to the recombination dynamics in the presence of a hole-conducting medium, process CR2 in Figure 1. We first consider recombination of electrons with a liquid $\mathrm{I}^{-} / \mathrm{I}_{3}{ }^{-}$ redox electrolyte. Figure 5 compares the transient absorption kinetics of complete liquid electrolyte cells as a function of sensitizer dye. Data are shown for a probe wavelength of 1000 $\mathrm{nm}$; at this wavelength, absorption of injected electrons $\left(\mathrm{e}^{-}{ }_{\mathrm{TiO} 2}\right)$ dominates the transient signal. ${ }^{36}$ These decay dynamics therefore primarily monitor the recombination of injected electrons with the redox electrolyte. As expected from literature data, ${ }^{6,37}$ these

(36) Green, A. N. M.; Palomares, E.; Haque, S. A.; Kroon, J. M.; Durrant, J. R. J. Phys. Chem. B 2005, 109, 12525-12533.

(37) Green, A. N. M.; Chandler, R. E.; Haque, S. A.; Nelson, J.; Durrant, J. R. J. Phys. Chem. B 2005, 109, 142-150. 


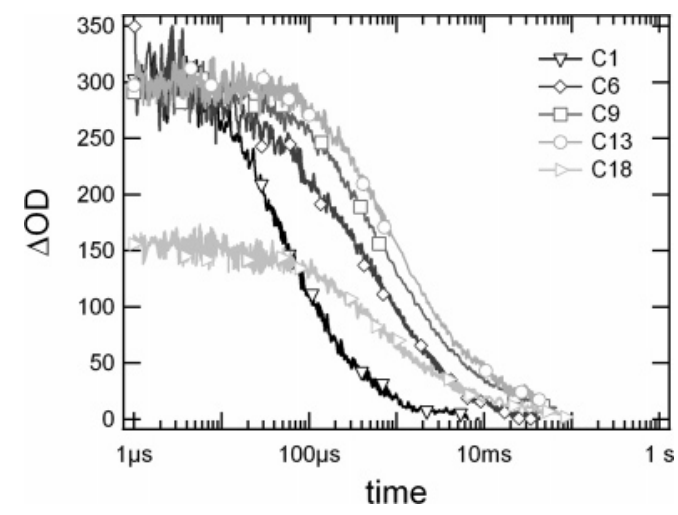

Figure 6. Transient absorption data monitoring electron recombination at $1000 \mathrm{~nm}$ in solid-state DSSCs employing the $\mathbf{C 1}-\mathbf{C 1 8}$ sensitizer dye series. The hole conductor matrix was applied by spin-coating from a chlorobenzene solution containing $0.17 \mathrm{M}$ spiro-OMeTAD, $25 \mathrm{mM} \mathrm{Li}^{+}, 0.13 \mathrm{M} t \mathrm{BP}$, and $0.3 \mathrm{mM}$ Sb dopant.

CR2 decay dynamics are approximately 2 orders of magnitude slower than the recombination to dye cations observed in the absence of a redox electrolyte (Figure 4). It is apparent from Figure 5 that we observe a retardation of the electron recombination kinetics with the increasing length of the dye alkyl chain spacers, with more than an order of magnitude slower recombination in the $\mathbf{C 1 8}$ dye-based cell compared to the $\mathbf{C 1}$ dye.

We have demonstrated previously that the hydrophobic alkyl chains of the present dye series are capable of providing increased spatial separation between electrons and cations on the solid-state hole conductor spiro-OMeTAD, resulting in retardation of the electron recombination kinetics and significantly increased device performance. ${ }^{25}$ Here we compare the transient absorption data of the dyes monitoring the electron recombination in a complete solid-state DSSC configuration, with the addition of $\mathrm{Li}^{+}, t \mathrm{BP}$, and $\mathrm{Sb}$ dopant to the hole conductor matrix. Transient absorption data monitoring at 1000 $\mathrm{nm}$ of the dye series in the solid-state device configuration are shown in Figure 6. In accordance with preceding investigations, for the $\mathbf{C 1}$ to $\mathbf{C 1 3}$ dyes closely similar injection yields are found, with the $\mathbf{C 1 8}$ deviating from the series. This reduced injection yield has previously been ascribed to inhomogeneous dye loading of the $\mathrm{TiO}_{2}$ surface due to the folding of the long $\mathbf{C 1 8}$ chains and was reflected in the significantly reduced device performance. As for the liquid-electrolyte device, upon increasing dye alkyl chain length a steady decrease of the electron recombination rate is observed, with an exception for the $\mathbf{C 1 8}$ dye, which shows marginally faster recombination compared to the C13 dye in the solid-state device configuration. We ascribe this, as before, to the incomplete swelling of the long C18 chains in the presence of spiro-OMeTAD, thereby reducing the recombination blocking effect of the alkyl chain spacer. ${ }^{25}$

The CR2 dynamics in the solid-state DSSCs shown here are more than an order of magnitude slower than our previously published recombination dynamics in the presence of spiroOMeTAD only. ${ }^{25}$ This in accordance with the proposed function of the common cell additives $\mathrm{Li}^{+}$and $t \mathrm{BP}$ of screening electrostatic interactions and shifting the density $\mathrm{TiO}_{2}$ conduction band/trap states, respectively, both resulting in retardation of interfacial charge recombination. ${ }^{38}$ Despite this, the CR2 charge

(38) Krüger, J.; Plass, R.; Cevey, L.; Piccirelli, M.; Grätzel, M.; Bach, U. Appl. Phys. Lett. 2001, 79, 2085-2087.

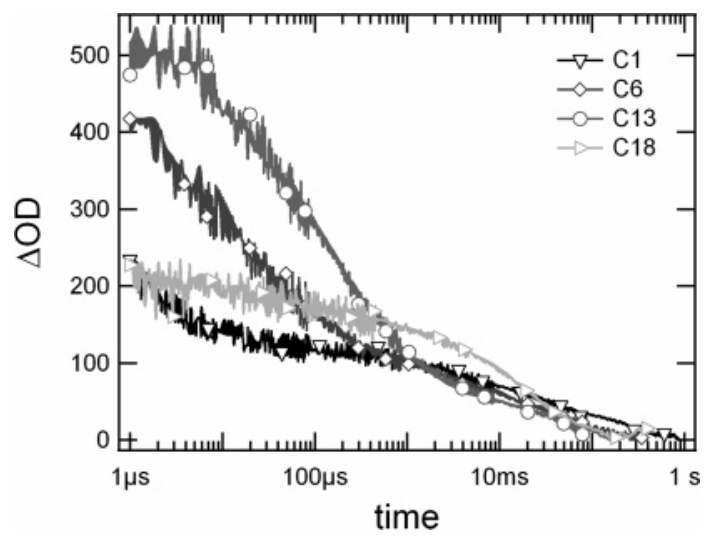

Figure 7. Transient absorption data monitored at $775 \mathrm{~nm}$ of dyes C1, C6, C13, and $\mathbf{C 1 8}$ in complete liquid $\mathrm{I}^{-} / \mathrm{I}_{3}{ }^{-}$redox electrolyte cell configuration, following $532 \mathrm{~nm}$ excitation.

recombination dynamics of the solid-state device are approximately 2 orders of magnitude faster than those observed for analogous liquid-electrolyte devices, consistent with our previous observations with the $\mathrm{Ru}(\mathrm{dcbpy})_{2}(\mathrm{NCS})_{2}$ sensitizer dye. ${ }^{19}$ We will discuss this in more detail in a following section.

Dye Regeneration (RG). We first consider the dynamics of the regeneration of the dye ground state by hole transfer from the dye cation to the liquid $\mathrm{I}^{-} / \mathrm{I}_{3}{ }^{-}$redox electrolyte. Figure 7 displays transient absorption data monitored at $775 \mathrm{~nm}$ for the C1 to $\mathbf{C 1 8}$ sensitizer dye cations in complete liquid electrolyte DSSCs. For the $\mathbf{C 1}$ and $\mathbf{C 6}$ dyes, two distinct regimes in the decay dynamics are observed, analogous to those we have reported previously for the $\mathrm{Ru}(\mathrm{dcbpy})_{2}(\mathrm{NCS})_{2}$ dye. ${ }^{27}$ The fast phase (microseconds) is assigned as previously to regeneration of the dye ground state from the dye cation by reduction by $\mathrm{I}^{-}$ with the concomitant generation of $\mathrm{I}_{2}^{-}$radicals, whereas the residual long-lived (millisecond lifetime) signal reflects the absorption of these $\mathrm{I}_{2}{ }^{-}$radicals and the concomitant absorption of long-lived electrons. ${ }^{39}$ Decay of this long-lived signal is assigned to $\mathrm{I}_{2}{ }^{-}$dismutation and recombination with $\mathrm{TiO}_{2}$ electrons (process CR2 in Figure 1). It is apparent that increasing the chain length results in a retardation of the regeneration reaction by over 2 orders of magnitude. For the $\mathbf{C 1 3}$ and particularly $\mathbf{C 1 8}$ dyes, the regeneration reaction is retarded to such an extent that it is no longer kinetically resolved from the slow phase assigned to CR2.

In sharp contrast to the liquid-electrolyte DSSCs, the dynamics of regeneration of the dye ground state by spiro-OMeTAD, in the presence of the standard solid-state cell components $\mathrm{Li}^{+}$, $t \mathrm{BP}$, and $\mathrm{Sb}$ oxidant, were found to be essentially complete within the instrument response of our microsecond-millisecond laser system for all sensitizer dyes. Further experiments employing nanosecond spectroscopy (see Supporting Information) indicated that, for dyes $\mathbf{C 1 - C 9}$, dye ground state regeneration was complete within $<<10$ ns, while, for dyes $\mathbf{C 1 3}$ and $\mathbf{C 1 8}$, a rapidly decaying transient was observed indicative of a regeneration half time of approximately $10 \mathrm{~ns}$. These observations are in agreement with our previous ultrafast study of charge transfer from spiro-OMeTAD to $\mathrm{Ru}(\mathrm{dcbpy})_{2}(\mathrm{NCS})_{2},{ }^{29}$ where hole transfer from the dye cation to spiro-OMeTAD was found to occur on timescales ranging from picoseconds to several

(39) Nogueira, A. F.; De Paoli, M. A.; Montanari, I.; Monkhouse, R.; Nelson, J.; Durrant, J. R. J. Phys. Chem. B 2001, 105, 7517-7524. 
Table 2. Device Performance Parameters for Liquid-Electrolyte and Solid-State DSSC Employing the C1-C9 Sensitizer Dye Series $^{a}$

\begin{tabular}{|c|c|c|c|c|c|c|c|c|}
\hline \multirow[b]{2}{*}{$\begin{array}{c}\text { sensitizer } \\
\text { dye }\end{array}$} & \multicolumn{4}{|c|}{ liquid-electrolyte device } & \multicolumn{4}{|c|}{ solid-state device ${ }^{c}$} \\
\hline & $\begin{array}{c}J_{\mathrm{sc}} \\
\left(\mathrm{mA} / \mathrm{cm}^{2}\right)\end{array}$ & $\begin{array}{c}V_{o c} \\
(\mathrm{mV})\end{array}$ & $\begin{array}{l}\mathrm{FF} \\
(\%)\end{array}$ & $\begin{array}{c}\eta \\
(\%)\end{array}$ & $\begin{array}{c}J_{\mathrm{sc}} \\
\left(\mathrm{mA} / \mathrm{cm}^{2}\right)\end{array}$ & $\begin{array}{c}V_{o c} \\
(\mathrm{mV})\end{array}$ & $\begin{array}{l}\mathrm{FF} \\
(\%)\end{array}$ & $\begin{array}{c}\eta \\
(\%)\end{array}$ \\
\hline C1 & 6.2 & 660 & 56.4 & 2.4 & 5.4 & 714 & 59.7 & 2.3 \\
\hline C6 & 6.4 & 690 & 57.9 & 2.6 & 5.8 & 712 & 60.5 & 2.5 \\
\hline $\mathbf{C} 9^{b}$ & & & & & 6.3 & 738 & 61.3 & 2.8 \\
\hline C13 & 7.0 & 750 & 61.6 & 3.2 & 6.3 & 744 & 66 & 3.1 \\
\hline C18 & 3.5 & 670 & 56.1 & 1.3 & 5.8 & 718 & 55.2 & 2.3 \\
\hline
\end{tabular}

${ }^{a}$ Data represent the average values of three devices. ${ }^{b}$ Due to problems associated with the adhesion of the $\mathbf{C 9}$ dye to the $\mathrm{TiO}_{2}$ surface, no reliable data for this dye can be given. ${ }^{c}$ Taken from ref $25 .{ }^{d}$ Note that in order to maintain compatibility with the transient absorption measurements, for the liquid-electrolyte devices thin, nonscattering $\mathrm{TiO}_{2}$ films have been used.

nanoseconds, i.e., more than 3 orders of magnitude faster than regeneration by the liquid-electrolyte redox species. Consistent with these fast regeneration dynamics, the yield of dye regeneration, determined as previously ${ }^{32}$ from the magnitude of dye cation absorption in the presence and absence of spiroOMeTAD, was $>90 \%$ for all dyes, confirming that even for the longer alkyl chain dyes the regeneration reaction competes effectively charge recombination to the dye cation, CR1. We present liquid-electrolyte and solid-state device characteristics of devices employing the different amphiphilic sensitizer dyes in the following section.

Device Characterization. Table 2 summarizes the photovoltaic performance characteristics of liquid-electrolyte and solid-state devices employing the different amphiphilic sensitizer dyes. Note that, in order to make transient optical absorption measurements possible, for the liquid-electrolyte devices optically transparent, thin $(\sim 4 \mu \mathrm{m}) \mathrm{TiO}_{2}$ films were applied, different from the scattering, thicker $(\sim 10 \mu \mathrm{m})$ films commonly applied for liquid-electrolyte DSSC fabrication. This will reduce the amount of incident light that is absorbed, resulting in reduced values for $J_{\mathrm{sc}}$.

In general, we observe for both types of devices an increase of all device parameters with increasing alkyl chain length of the sensitizer, up to the $\mathbf{C 1 3}$ dye, which displays the best performance in both the liquid-electrolyte and solid-state cell configuration. In the following section we discuss the correlation between the dynamics and kinetic redundancy of the interfacial charge-transfer steps and liquid-electrolyte and solid-state device performance.

\section{Discussion}

We have previously shown that optimum DSSC performance in terms of the electron injection dynamics is obtained if the injection kinetics are just fast enough to compete with the excited-state decay of the dye. ${ }^{23}$ Such optimized kinetics were achieved by the use of $\mathrm{Li}^{+}$ions and $t \mathrm{BP}$ additives in the electrolyte to modulate the energetics of the $\mathrm{TiO}_{2}$ conduction band. More specifically, although omission of $t \mathrm{BP}$ was found to result in fast electron injection kinetics, this process was found to occur at the expense of fast recombination dynamics, and hence low $V_{\mathrm{oc}}$. Conversely, omission of $\mathrm{Li}^{+}$ions resulted in slow recombination yet was paralleled by slow injection and low $J_{\text {sc }}$. This led us to the conclusion that device optimization requires a suitable compromise between the requirements of high electron injection yields and slow recombination and the avoidance of any redundancy in the kinetics of the electron injection process. Such considerations are however not restricted to the electron injection process only but most likely apply to all charge-transfer steps occurring in photovoltaic devices.

In the present study we have extended our studies to a series of amphiphilic ruthenium dyes, derivatives of the recently reported $\mathbf{Z 9 0 7}$ dye (this dye being the $\mathbf{C 9}$ dye employed in the present study). ${ }^{10}$ This $\mathbf{Z 9 0 7}$ dye is a derivative of the $\mathbf{N 7 1 9}$ dye, which is at present widely used in solid-state and liquidelectrolyte device preparation. Both dyes have shown superior performance in terms of efficiency of the resulting DSSC. ${ }^{10,26}$

We first of all consider the electron injection dynamics we observe for the $\mathbf{C 1 - C 1 8}$ dye series. Data are reported for dyesensitized $\mathrm{TiO}_{2}$ films covered in inert solvent and redox electrolyte and compared against control data for noninjecting dye-coated $\mathrm{ZrO}_{2}$ films. As expected, electron injection dynamics are observed to be approximately 1 order of magnitude slower in the presence of redox electrolyte compared to inert solvent, assigned, as we have discussed previously, ${ }^{23}$ to the influence of the redox electrolyte on the $\mathrm{TiO}_{2}$ conduction band energetics. Less expected is our observation that the electron injection dynamics for this dye series extend to significantly longer time scales than those we observe for the $\mathbf{N 1 9}$ dye. The C1 dye exhibits the fastest dynamics: $<60 \mathrm{ps}$ in the presence of the redox-inactive electrolyte and 625 ps in the redox electrolyte. Increasing the alkyl chain length up to $\mathbf{C 9}$ results in a progressive retardation of dynamics. For all dyes except C18, the injection dynamics for the $\mathrm{TiO}_{2}$ films are at least four times faster than those for the control $\mathrm{ZrO}_{2}$ films, consistent with a high yield of electron injection into $\mathrm{TiO}_{2}$. We furthermore note that the excited-state decay dynamics observed on $\mathrm{ZrO}_{2}$ were collected under aerobic conditions and therefore represent an upper (fastest) limit for the rate of excited-state decay to the ground state in a sealed, and therefore anaerobic, DSSC; hence, even slower dynamics may be expected for $\mathrm{ZrO}_{2}$ under the anaerobic conditions present in sealed DSSCs.

The retardation of emission decay dynamics with increasing alkyl chain length up to $\mathbf{C 9}$ is surprising. One possible explanation for this trend is an increased spatial separation of the dye LUMO orbital occupied in the dye excited state from the $\mathrm{TiO}_{2}$ surface. This could result from the influence of the alkyl chains upon the orientation of the dye adsorbed to the surface. We note that this dye series contains only a single bi(carboxypyridine) (dcbpy) group responsible for anchoring the dye to the $\mathrm{TiO}_{2}$ substrate (compared to two such groups in the N719 dye), consistent with greater flexibility in binding orientation (see also below). Such a reorientation effect and/or wrapping of the Ru center with aliphatic chains could account for an increase in irradiative processes as well as the retardation of the CR1 recombination reaction with increasing alkyl chain lengths (see Figure 4). However, a strong dependence of electron injection upon dye orientation would only be expected if the dye excited state LUMO orbital were localized not on the dcbpy but rather upon on the bipyridine group attached to the alkyl chain moieties (dabpy). Previous LUMO orbital calculations for the isolated $\mathbf{C 1}$ dye have however indicated a small energetic stabilization of the LUMO orbital localization upon the dcbpy 

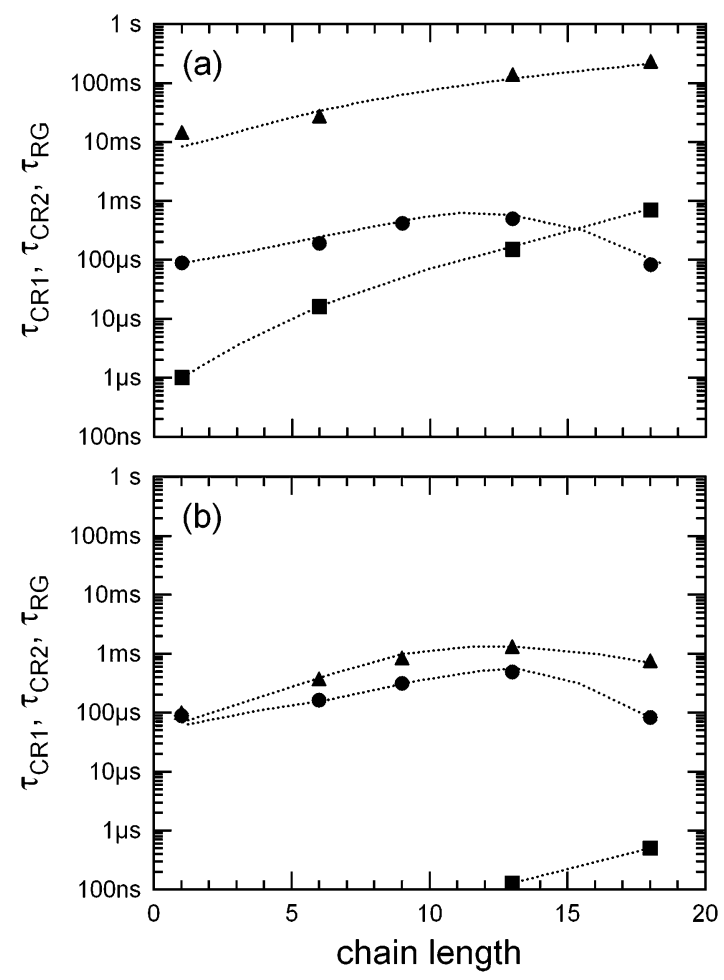

Figure 8. Half times of the interfacial electron-transfer reactions (see Figure 1) CR1 $(\bullet), C R 2(\boldsymbol{\Delta})$, and RG (অ), as a function of the alkyl chain length for (A) liquid-electrolyte and (B) solid-state dye-sensitized solar cells.

moiety, ${ }^{40}$ although we note that this LUMO localization may be significantly modified by interface dipoles present at the $\mathrm{TiO}_{2} /$ electrolyte interface. We emphasize that a 100-fold retardation of the electron injection rate only requires a $5 \AA$ increased spatial separation. Alternatively, it is possible that the alkyl chain groups modulate the energetics of electron injection. All of these dyes $\mathbf{C 1}-\mathbf{C 1 8}$ exhibit similar excited-state oxidation potentials $E\left(\mathrm{D}^{+} / \mathrm{D}^{*}\right)=-0.94 \pm 0.02 \mathrm{~V}$ vs SCE (more negative than the equivalent potential for N719, $-0.68 \mathrm{~V}$ ), determined from solution electrochemical and optical data. We note, however, that the presence of the alkyl chains has been shown to significantly reduce the extent of hydration of the $\mathrm{TiO}_{2}$ surface. ${ }^{24}$ Immersion of mesoporous $\mathrm{TiO}_{2}$ films in anhydrous solvents has previously been shown to result in large (negative) shifts in the conduction band edge relative to aqueous environments, ${ }^{41}$ suggesting that the inclusion of the alkyl chains on the dye may significantly reduce the free energy driving force $\Delta G$ for electron injection.

We now consider the dynamics of charge recombination and dye regeneration by the $\mathrm{I}_{2} / \mathrm{I}^{-}$redox couple in liquid-electrolyte DSSCs. Figure 8A summarizes the characteristic half times for charge transfer in the liquid-electrolyte device as a function of the sensitizer alkyl chain length. Consistent with the proposed blocking function of the hydrophobic alkyl chains, we find a retardation of the recombination kinetics upon increasing chain length of close to 2 orders of magnitude. We note that this trend is observed not only for recombination to the redox couple (CR1) but also to the dye cation (CR2), consistent with the suggestion above that increasing alkyl chain length results in the dye orientating more perpendicular to the $\mathrm{TiO}_{2}$ surface. As

(40) Nazeeruddin, M. K.; De Angelis, F.; Fantacci, S.; Selloni, A.; Viscardi, G.; Liska, P.; Ito, S.; Takeru, B.; Grätzel, M. G. J. Am. Chem. Soc. 2005 , $127,16835-16847$. anticipated, this is reflected in an increase of the device opencircuit voltage up to the $\mathbf{C 1 3}$ sensitizer.

Similar to our previous studies on the kinetics of electron injection vs recombination as a function of cell additives, we find that the retardation of the electron recombination dynamics with increasing alkyl chain length and the corresponding dye regeneration kinetics are correlated. As predicted, we observe that the regeneration reaction $(\mathrm{RG})$ rapidly retards as the chain length is increased. However, we note that the RG dynamics are considerably more sensitive to the hydrophobic barrier layer thickness than the dynamics of the CR2 reaction, with barrier layer insertion resulting in an $\sim 20$-fold retardation of CR2 compared to an $\sim 700$-fold slower RG dynamics. This nonparallel retardation of the charge-transfer dynamics is readily explained by consideration of the electrolyte species involved in the CR2 and RG reactions. Recombination of photoinjected electrons proceeds through hydrophobic $\mathrm{I}_{2}$ molecules, whereas the dye regenerating species are the hydrophilic $\mathrm{I}^{-}$ions, which are considerably stronger hindered by the hydrophobic alkyl chain barrier. For chain lengths greater than $\mathbf{C 1 3}$, the regeneration reaction $\mathrm{RG}$ is actually slower than recombination to the dye cation (CR1), consistent with the reduced $J_{\mathrm{sc}}$ of C18sensitized devices.

The half times for the charge-transfer reactions in solid-state spiro-OMeTAD-based DSSCs are summarized in Figure 8B. Similar to the liquid-electrolyte devices, the progressive retardation of the electron-hole recombination process CR2 due to the blocking effect of the alkyl chain lengths up to $\mathbf{C 1 3}$ is reflected in the parallel increase in device performance parameters. For these devices, the dynamics of the recombination reactions $\mathrm{CR} 1$ and $\mathrm{CR} 2$ are of similar magnitude, as we have discussed in detail elsewhere. ${ }^{19}$ In fact, this localization of photogenerated species at the $\mathrm{TiO}_{2} /$ dye interface and the concomitant fast recombination dynamics form one of the underlying reasons for the reduced performance of solid-state DSSCs, as they limit the diffusion length of the photogenerated species in the device and, hence, restrain the maximum device thickness (and subsequent light absorption) compatible with efficient charge collection.

The dye regeneration ( $R G$ ) by spiro-OMeTAD is several orders of magnitude faster than by the liquid electrolyte, being $<10$ ns for dyes $\mathbf{C 1 - C 9}$ and still proceeding on the $10 \mathrm{~ns}$ time scale even for the $\mathbf{C 1 3}$ and $\mathbf{C 1 8}$ dyes. We conclude that in contrast to the analogous liquid-electrolyte cells, for the solidstate DSSCs, the regeneration reaction is 4 orders of magnitude faster than the competing recombination reaction CR2, even for longest alkyl chain dye C18. In other words, the chargetransfer dynamics in the present series of solid-state devices are still "kinetically redundant" with respect to the regeneration reaction. We emphasize that, in contrast to the liquid-electrolyte cells, the reduced solid-state cell performance of the C18-dyebased device cannot be caused by kinetic competition of the interfacial charge-transfer reactions, as is obvious from Figure $8 \mathrm{~B}$, but is most likely due to problems associated with the collapse of the long alkyl chains, resulting in a reduced electron injection yield and incomplete wetting by spiro-OMeTAD.

Nevertheless, our results demonstrate that inhibition of the $\mathrm{CR} 2$ recombination reaction is a key point in the optimization

(41) Redmond, G.; Fitzmaurice, D.; Grätzel, M. J. Phys. Chem. 1993, 97, 69516954 
of solid-state devices. Besides the present strategy of blocking alkyl chains, these interface engineering approaches may include the extension of the anchoring groups of the dye, the molecular level insulation of redox-active species,${ }^{42}$ and the use of multiple hole-conducting layers differing in ionization potential. Such an organic redox cascade has recently demonstrated the ability to increase the spatial separation of photogenerated electrons and holes, resulting in a significantly reduced electron-hole recombination. ${ }^{19}$ In view of our previous work ${ }^{23}$ and the results shown in this paper, such approaches will invariably also affect the yields and dynamics for dye regeneration and interfacial electron injection.

More generally, we further note that finding "optimum" charge-transfer dynamics in nanostructured DSSCs may be impeded by local inhomogeneities caused by the dispersive nature of the $\mathrm{TiO}_{2}$ and interfacial electrostatic interactions, causing highly dispersive charge-transfer dynamics. ${ }^{32,43}$

\section{Conclusions}

We have studied the charge separation and recombination dynamics in liquid-electrolyte and solid-state dye-sensitized solar cells. More specifically, we have addressed the extent of redundancy present in the dye regeneration dynamics and determined the "optimum" charge-transfer dynamics for the best possible device function.

We have found that efficient retardation of electron-hole recombination in both liquid-electrolyte and solid-state cells can be achieved by the application of recombination-blocking amphiphilic alkyl chains attached to the sensitizer dye, resulting in a significantly improved device performance. For the liquidelectrolyte devices, however, the dye cation recombination dynamics exceed the dye regeneration speed for the longest alkyl chains, which is reflected in the reduced device performance.

Such "optimized" interfacial dynamics are not observed for the solid-state device analogues, which are still kinetically redundant in terms of regeneration dynamics. In these cells, however, the dynamics of electron recombination with the dye cation and the hole conductor are within the same order of

(42) Haque, S. A.; Park, J. S.; Srinivasarao, M.; Durrant, J. R. Adv. Mater. 2004 16, $1177-1181$

(43) Kroeze, J. E.; Hirata, N.; Schmidt-Mende, L.; Orizu, C.; Ogier, S. D.; Carr K.; Grätzel, M.; Durrant, J. R. Adv. Funct. Mater. 2006, 16, 1832-1838. magnitude, suggesting the lack of sufficient spatial separation of the photogenerated charges.

We find that the addition of alkyl chains to the sensitizer dye results in a significant retardation of dynamics to the redox electrolyte/spiro-OMeTAD but also of the dynamics of charge injection and recombination between the sensitizer dye and the $\mathrm{TiO}_{2}$ surface. This retardation is consistent with different binding orientations of the sensitizer dye, with the longer alkyl chains resulting in the sensitizer dye being orientated more normal to the $\mathrm{TiO}_{2}$ surface.

In the study we report here, minimization of kinetic redundancy in the device is achieved by barrier layer insertion. We note that several other strategies employed to optimize device performance can also be viewed as strategies designed to minimize kinetic redundancy in the device. These strategies include optimization of the sensitizer dye protonation, ${ }^{44}$ modulation of the hole conducting medium composition, ${ }^{19}$ and the coating of the nanocrystalline $\mathrm{TiO}_{2}$ film with metal oxide barrier layers, ${ }^{4,5,7,9}$ with the barrier layer thickness being optimized to still allow efficient electron injection while at the same time minimizing interfacial recombination dynamics. Such "interface engineering" strategies directed toward the control of such electron-transfer dynamics at nanostructured inorganic/organic interfaces are the subject of ongoing research in our labs.

In summary, the optimization of nanostructured solar cells eventually depends on achieving a careful balance between the constraints of fast charge separation and slow recombination and the reduction of any kinetic redundancy.

Acknowledgment. We acknowledge funding from the European Commission (FP6 MOLYCELL project, Contract No. 502783) and the EPSRC Excitonic Solar Cells Supergen program.

Supporting Information Available: Nanosecond transient absorption data monitoring regeneration of the dye ground state of the C9, C13, and C18 dye in complete solid-state device configuration; non-normalized transients of Figures 4 and 5. This material is available free of charge via the Internet at http://pubs.acs.org.

JA065653F

(44) Tachibana, Y.; Nazeeruddin, M. K.; Grätzel, M.; Klug, D. R.; Durrant, J. R. Chem. Phys. 2002, 285, 127-132. 